\section{Imaging with the \\ Photon Scanning-Tunneling Microscope}

Thomas L. Ferrell, Fabrice Meriaudeau, Ali Passian, Jean-Pierre Goudonnet, and Andrew Wig Oak Ridge National Laboratory

\section{INTRODUCTION}

The use of traditional optics in microscopy has a well-known resolution barrier first presented by Lord Rayleigh. This is the "Rayleigh diffraction limit." In the usual textbook example, the overlapping diffraction rings from two small objects are set so that the central maximum of one pattern falls on the first minimum of the second. The barrier limits the resolution of conventional microscopes to a significant fraction of the wavelength of light.

In the past decade two methods were discovered for surpassing the Rayleigh diffraction barrier. The first was actually an independent rediscovery by D. Pohl et al. ${ }^{1}$ of a concept originally proposed by Synge ${ }^{2}$ (in a 1928 paper reviewed by Albert Einstein). This form of scanning-probe microscopy is termed "near-field scanning optical microscopy" (NSOM). The second follows the same physical principle as the famous electron scanning-tunneling microscope (STM) and is called the "photon scanning-tunneling microscope" (PSTM). ${ }^{3}$

In the NSOM, a sharp, transparent probe (normally an optical fiber) is coated with an opaque coating (usually aluminum) except for an aperture at the apex. The aperture is of a size much smaller than the wavelength of the light used. Interestingly, light can enter or exit the aperture despite its small size. It essentially acts as an antenna. Light can be transmitted through the sample and detected at very close distances. In this case, and in the case in which the probe sends light to be reflected back, the sample is actually radiating (or reradiating). In classical electrodynamics, when an observer in a radiating object's radiation field is within a fraction of a wavelength of the object, the observer is said to be in the "near zone". Conventional microscopes use the intermediate zone or far zone. By scanning the probe near the sample, an image can be formed from the resulting variation in signal intensity. A "shear force" attachment (measures force between the sample and tip) is used to attempt to determine how close the probe is to the sample. Resolution is determined by the electrodynamical size of the aperture and the characteristics of the sample.

Following the discovery of the STM by $G$. Binnig and $H$. Rohrer ${ }^{4}$, a new type of photon-based scanning-probe microscope was invented using essentially the same principle as the STM. In this case, the famous de Broglie relationship (momentum proportional to wavelength) and the wave behavior of particles in quantum mechanics was the basic concept used for the discovery. It was known, for example, that the tunneling coefficient for s-polarized photons (linearly polarized perpendicular to the plane of incidence) and for electrons is basically the same under analogous conditions. The PSTM and the STM obtain high-resolution due to the very strong exponential dependence of the tunneling phenomenon. In scanning-tunneling microscopes the probe is very sharp. Hence, any region away from the apex is also farther from the sample and thus receives exponentially less flux. Instead of seeking to confine the signal with the probe, the signal is obtained only from a limited area of diameter much less than the wavelength of the tunneling photons. Also, the signal has a definitive dependence upon distance of the probe from the sample so that a shear-force attachment is not needed. In fact, the distance to the surface is the wavelength multiplied by the negative of the derivative of the distance with respect to wavelength. Most noteworthy is that a PSTM sample is nonradiative (in the direction of the observer), and strictly speaking there is no "near zone" in this case. In fact, the exponential function simply continues its decrease as one moves away from the sample as opposed to the complicated spatial dependence found for radiating objects. Of course, the tip must be very close to the sample in practice.

The NSOM is capable of imaging thick samples that are opaque to the available wavelengths, but it has difficulty with transparent objects. The PSTM is capable of imaging samples which are thin or which are transparent to totally internally reflected light (see Figure 1) at one or more wavelengths in the ultraviolet to infrared region of the spectrum. (Metals, for example, become transparent in the ultraviolet.) In PSTM imaging, the photons are initially made to undergo total internal reflection within the sample. Thus, strongly absorbing samples can be imaged by the PSTM at no more than a few wavelengths thickness. In a sense, the NSOM optically "feels" the sample in an analogy to the atomic force microscope (AFM). There is no consistent dependence of signal upon the probesample gap, while the PSTM is more like the STM in that it relies upon the welldefined exponential signal of tunneling. For spectroscopic purposes, the PSTM is often employed since the intensity of the signals is not reduced by the necessity of passing photons through an aperture.

The physical topography of a sample is best sensed by an atomic force microscope as the PSTM measures the optical topography, the STM measures the electronic topography, and the NSOM measures optical response topography (scattering or transmission response). But all of these provide three-dimensional data.

Figure 1 shows a schematic of a typical PSTM setup.

\section{BACKGROUND}

It may be valuable to consider some general background at this point. Tunneling in classical optics has long been known as frustrated total internal reflection. Light waves are totally internally reflected in matter bounded by vacuum (or by matter having a lower index) if the angle of incidence at the surface is greater than the critical angle. But electrons also behave like waves in quantum theory. Tunneling commences if a probe is placed well within a wavelength of the surface. For free electrons of a metal impinging on the surface, the electrons are totally internally reflected at angles greater than the critical angle (which depends upon temperature and the characteristics of the metal). If an external electric field is applied by a nearby electrode, the electrons can tunnel out of the metal. That is, they escape despite the fact that their kinetic energy is classically insufficient relative to the potential energy barrier at the surface (related to the "work function"). In the case of photons inside matter, say quartz, the photons are totally internally reflected for angles of incidence above the critical angle. In both cases, the wavefunction for total internal reflection actually extends beyond the boundContinued on Page 16

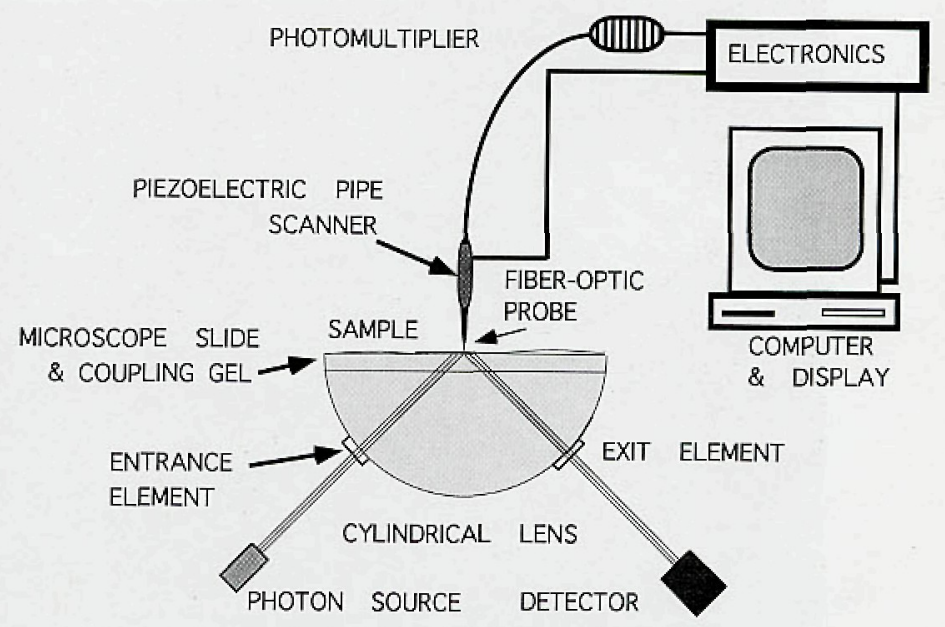

Figure 1. Schematic of the PSTM. Photons are directed into the cylindrical lens through an entrance element that prevents focusing. The angle of incidence of photons on sample is set at just above the critical angle so that total internal reflection occur i.e., no light exits through the sample via refraction towards the probe. Then the probe (a very sharp optical fiber) is brought to within a fraction of a wavelength distance from the sample or until the exponential tunneling sigrial is observed to be engendered by the local frustration of the internal reflection. The probe is scanned across the sample and tracks the exponential variations in signal at constant distance or it may be adjusted to move in or out so as to maintain a constant current by use of feedback circuitry. 

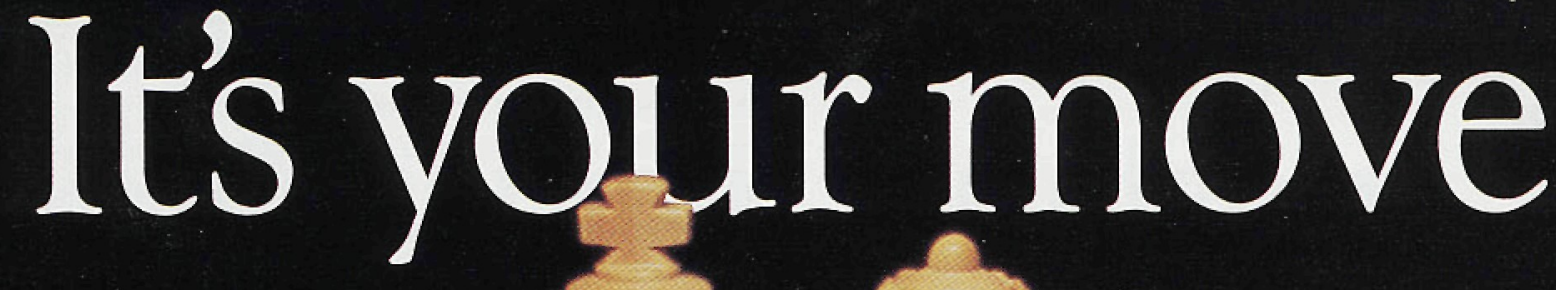

ThermoMicroscopes offers more choices for nanoscale visualization than anyone. That means you choose the best scanning probe microscope for your application. Consider...

\section{VIMTHERMOMICROSCOPES} ¿ङPark Scientific Instruments KTOPOMETRIX

1171 Borregas Avenue, Sunnyvale, CA 94089 Telephone 408.747.1600 Fax 408.747.1601 info@thermomicro.com

\section{Explorer}

The most versatile SPM ever built. Scans samples of any size. Dedicated system solutions for polymer and life sciences.
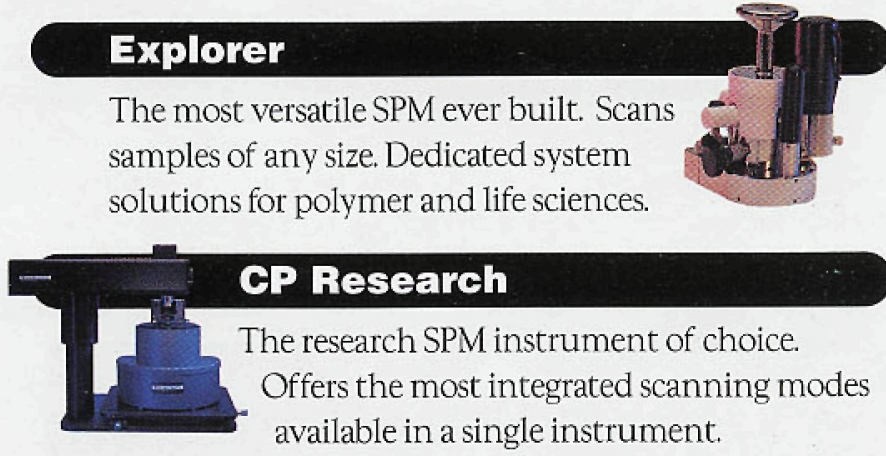

\section{Autoprobe M5}

An analytical instrument designed for industrial applications. Advanced SPM

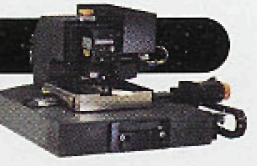
technology at an affordable price.

\section{Aurora NSOM}

$B$ Designed and built by the worlds leading manufacturer of NSOM systems. Features patented tuning-fork technology.

And those are just our opening moves. We've got more. Lot's more. So consider your choices carefully.

Then make your move to www.thermomicro.com.

\section{The Differenge You Gan Meaguae}




\section{Imaging with the Photon Scanning-Tunneling Microscope \\ Continued from page 14}

ary with exponentially decreasing amplitude, but no flux is transmitted. The "index of refraction" for electrons and photons in plane-wave states can be defined as the ratio of momentum in matter relative to the momentum in vacuum. The critical angle is then just the arcsine of the reciprocal of the relative index of refraction $n$ of the sample and the bounding medium.

$$
\text { Critical angle }=\text { Arcsin }(1 / n)
$$

As a probe is brought within a fraction of a wavelength of a surface in which there is total internal reflection, the tunneling commences with a wellknown profile. The tunneling coefficient for plane waves incident on a plane boundary and tunneling into a second plane-bounded medium is not complicated. For both electron tunneling and the tunneling of s-polarized photons, the coefficient gives the ratio of intensity transmitted to the intensity incident upon the surface. There is a different coefficient for the case of p-polarized photons (linearly polarized within the plane of incidence).

As soon as the PSTM tunneling gap exceeds a nanometer or so, the tunneling signal becomes a simple decreasing exponential. The working distance of the PSTM lies within a distance ranging from $10 \mathrm{~nm}$ to $100 \mathrm{~nm}$ and the signal drops by factors of ten as the gap is increased relative to the wavelength. Of course, the scales for the STM are 500 times smaller as the wavelength of electrons (on the order of one $\mathrm{nm}$ ) is much less than that of photons (on the order of $500 \mathrm{~nm}$ ), so the spatial resolution of the STM is far superior for samples that it can examine. However, the spectroscopic resolution of photons is well over a million times better than can be obtained with electrons or with the various forms used in atomic force microscopy. This allows the PSTM to function well as an imaging spectrometer. ${ }^{5}$ To date, spectroscopies carried out with the PSTM include optical absorption, infrared absorption, surface-enhanced Raman, and fluorescent spectroscopy. 6,7

In a later article the spectroscopic capabilities of the PSTM will be examined.

\section{PSTM IMAGING}

Figure 2 shows a PSTM image of pores in polymethylmethacrylate electron resist. These are produced by electron-beam lithography and each pore is $60 \mathrm{~nm}$ in diameter. The scale bar is $100 \mathrm{~nm}$ long, but the scale error in this

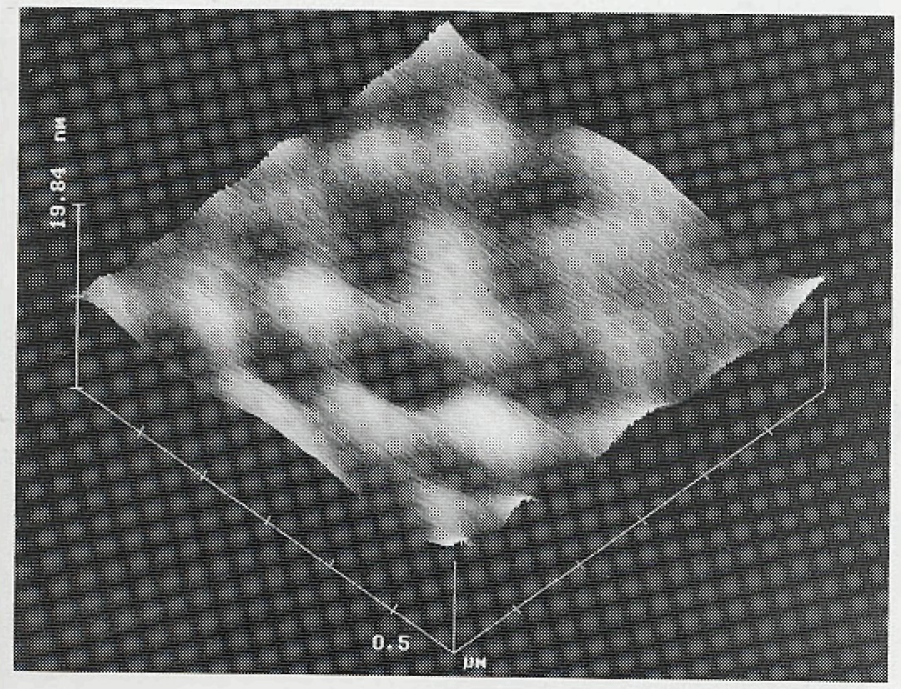

Figure 2. A PSTM image of $60 \mathrm{~nm}$ pores in electron resist. The bar is $100 \mathrm{~nm}$ in length with an error of plus or minus $10 \mathrm{~nm}$. case is plus or minus $10 \mathrm{~nm}$ since calibration on this scale is difficult. A standarch reference sample would provide better results, but was not available at the time Images at this resolution require a degree of flatness for the sample. The resolu. tion depends upon how close the probe can be placed during the scan without crashing into protrusions as it is rastered. In many cases the roughness features will cause the scanning probe to collide with the sample before it can be re- $\overrightarrow{0}$ tracted. The difficulty is not yet well quantified since it depends upon the re-w sponse of the particular commercial scanner used and we have limited experi-o ence in this regard to date. The difficulty is also apparent with other scanned of probes and the result is that the average distance of the probe from the sample? must be increased. This results in poorer resolution. Improvements in scanning $\frac{\bar{n}}{\overline{0}}$ mechanisms and in procedures are continually being made to address this prob-市 lem. Resolution remains an open question due to the dependence upon the sample and instrumentation, but the salient features of the PSTM are that it provides? $3 \mathrm{D}$ optical signals in air at high resolution for samples which are transparent (or $\frac{\hat{\Omega}}{3}$ thin), and it can perform many types of optical spectroscopy.

A collection of PSTM images, including images of biological interest such as chromosomes and liposomes, can be found on the world-wide web at: http://uww.sim-pstm.com/atechnologies_jmages.htm.

1. D.W. Pohl, W. Denk, and M. Lanz, Appl. Phys. Lett. 44, 651(1984)

2. F. H. Synge, Phil. Mag. 6, 356 (1928)

3. R. C. Reddick, R. J. Warmack, and T. L. Ferrell, "A New Form of Scanning Optical Microscopy," Phys. Rev. B 39, 767-770 (1989)

4. G. Binnig and $H$. Rohrer, "Scanning Tunneling Microscopy," Helv. Phys. Acta. 55, 726-735 (1982)

5. M. A. Paesler, P. J. Moyer, C. J. Jahncke, C. E. Johnson, R. C. Reddick, R. J. Warmack, and T. L. Ferrell, "Analytical Photon Scanning Tunneling Microscopy," Phys Rev. B 42(10), 6750-6753 (1990)

6. P. J. Moyer, C. L. Jahncke, M. A. Paesler, R. C. Reddick, and R. J. Warmack, "Spectroscopy in the Evanescent Field with an Analytical Photon Scanning Tunneling Microscope," Phys, Lett. A 145(6,7), 343-347 (1990)

7. T. L. Ferrell, S. L. Sharp, and R. J. Warmack, "Progress in Photon Scanning-Tunneling Microscopy (PSTM)," Uitramicroscopy 42-44, 408-15 (1992).

The submitted manuscript has been authored by a contractor of the U.S. Government under contract no. DE-AC05-960R22464. Accordingly, the U.S. Government retains a non exclusive, royalty-free license to publish or reproduce the published form of this contribution or allow others to do so, for U.S. Government purposes.

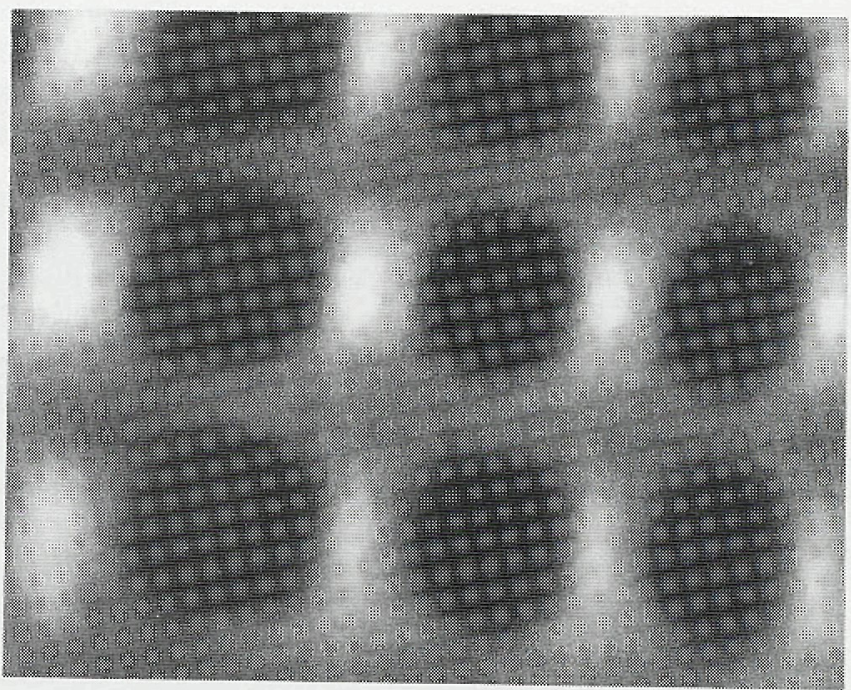

Figure 3. A gold-island film imaged by the PSTM using photons from a HeNe laser at a wavelength of $633 \mathrm{~nm}$. The vertical scale in this case may not be accurate for the physical topography due to the fact that the optical index of gold differs from that of the quartz substrate, but the particles are $5 \mathrm{~nm}$ in thickness. 


\section{Digital Productivity vs Darkroom Drudgery}

Advar tage Series TEM Camera Systems

- 1024 or 2048 Pixel Resolution

- Lens or Fiber Optic Coupling

- Large Field of View

- PC Based Turnkey Systems

- Standard TIFF File Format

- Axial or Side Mount Systems

- Up to 4096 Gray Levels

- Fast Acquisition Speeds

Advanced Microscopy Techniques Corporation

3 Electronics Avenue Danvers, MA 01923

Tel: (978)774-5550 Fax: (978)739-4313

Email: amtcorp@delphi.com

WWW: http://www.msa.microscopy.com/ amt/ 